\title{
Management of road transport-induced air pollution in urban areas: A case study of Owerri, Nigeria
}

\author{
Onyeka JO ${ }^{1}$, Obi LE ${ }^{1, *}$ and Igiri VC ${ }^{2}$ \\ ${ }^{1}$ Department of Civil Engineering, Imo State University, Owerri. \\ 2 Directorate of Works \& Services, Imo State University, Owerri.
}

International Journal of Frontiers in Engineering and Technology Research, 2021, 01(01), 030-044

Publication history: Received on 01 April 2021; revised on 02 May 2021; accepted on 04 May 2021

Article DOI: https://doi.org/10.53294/ijfetr.2021.1.1.0044

\begin{abstract}
Environmental concerns have continued to be on the increase as human activities soar. One such environmental concern is air pollution. A major contributor to air pollution is the road transportation activities. This paper sets out to provide a management strategy for air pollution owing to road transportation in urban areas, with Owerri Nigeria as a case study. A field study was conducted in Owerri to ascertain the total passenger requirement, number and mix of passenger vehicles as well as measure three main road transport-induced air pollutants at five locations in the city. The result of the field work showed existing commuter vehicles mix of 56.2:63.7: 19.6:1.6:1 of salons, wagons, mini-buses, coaster buses and big buses respectively, of a total of 85,950 vehicles and air ambient pollutants level higher than the recommended standards. A new model was developed to achieve a remix of 10:33:53: 14:1 of same vehicle types and reduction in traffic volume and target air pollutants. The analyses show that mini-buses and coaster buses have advantage over salon cars, wagon vehicles and big buses in terms of traffic congestions and pollutants release into the environment. The two bus types could be said to have least pollutants release per passenger carried. An optimal vehicle remix, which gives higher priority to these buses have been shown to reduce congestion by $40 \%$, Carbon monoxide by 40\%, Nitrogen Dioxide by 50\% and Methane by 50\%. It therefore recommends that vehicular remix of 10:33:53: 14:1, for salon: wagon: mini-buses: coaster buses: big buses be adopted for Owerri commuters' transportation need. It concludes that governments should adopt economic instruments embedded in a "push and pull" strategy, leveraging on disincentive and incentive measures to skew road transportation to the use of mini and coaster buses as a deliberate means of reducing air pollution in cities.
\end{abstract}

Keywords: Road; Transport; Air; Pollution; Urban

\section{Introduction}

Roads are specially prepared land ways for vehicular and pedestrian movements. In its simplest form the road can be the natural surface. It can also be a modified surface using local materials. As traffic increases, the road can be expanded, and its surface stabilized with imported materials, which can further be surfaced to improve speed and comfort.

Road transportation on the other hand is movement using roads (paved or unpaved). It is a land-based transportation mode, and can be roughly grouped into the transportation of goods and transportation of people.

People are transported on roads, either in individual cars or in mass transit buses or coaches. Special modes of individual transport by road such as bicycle may also be locally available. There are also specialist modes of road transport for particular situations, such as ambulances. The road transportation system is the aggregate of all facilities

\footnotetext{
${ }^{*}$ Corresponding author: Obi LE

Department of Civil Engineering, Imo State University, Owerri.

Copyright (C) 2021 Author(s) retain the copyright of this article. This article is published under the terms of the Creative Commons Attribution Liscense 4.0.
} 
put in place, maintained and or operated for the movement of people and goods by road. These include fixed structures (roads), mobile units (motor vehicles) and the operators of these facilities. Generally, these the mobile units (automobiles), are powered by either petrol (gasoline) or diesel internal combustion engines. Such internal combustion engines are known to be major sources of outdoor air pollution, and traffic is the most notable source of air pollution in urban areas. The relentless motorization of society has entailed an increasing growth of vehicle emissions which impact negatively on urban air quality.

Air pollution is defined as "the presence of one or more contaminants in the atmosphere (such as dust, fumes, gas, mist, odors, smoke or vapor) in quantities, characteristics, and of duration such as to be injurious to human, plant or animal life or to property or which unreasonably interferes with the comfortable enjoyment of life and property" (Subramani, 2012).

Owerri is the capital city of Imo State of Nigeria which is growing outwards from the city centre. It sits at the intersection of roads from Port Harcourt, Onitsha, Aba, Umuahia, Okigwe and Orlu and at latitude 5o 28' 35.6“(5.4766o) North and longitudes 7o 1' 0.6" (7.01680) East and elevation of 75m above sea level, (Encyclopaedia Britanica, 2009).

Over the years the seat of state government offices, markets, schools, banks, other business offices have sprung up within the capital city of Owerri. The city is also fed with traffic from the main entrance arteries of Orlu, Onitsha, PortHarcourt, Aba, Mbaise and Okigwe roads. Private and public housing projects have developed and continued to develop outwards from the centre. The obvious implication of this development style and the strategic location of Owerri as the eastern heartland is that majority of the people in Owerri have reasons to commute to and fro the city centre from their respective abodes that are some distances from the centre. One of the consequences of this necessary movement is vehicular congestion with the attendant air pollution from vehicle exhaust emission.

Writing on "What You can Do to Reduce Pollution from Vehicles and Engines," the United States Environmental Protection Agency (US EPA) stated that driving less, driving wise, choosing fuel efficient vehicle, avoiding idling, optimizing home deliveries and using efficient lawn and gardening equipment are effective strategies, (US EPA, 2017). In their opinion these source curtailment strategies are better options for pollution control management. The Energy Commission of Nigeria in the National Energy Policy (2003) in their opinion noted the need for cost effective strategies that will cut down on the demand for oil products and minimize environmental degradation arising from energy consumption, in the transportation sector. The Commission also stated that pollution is a major concern and that combustion of fossil fuels especially in the transportation and industrial sectors contribute greatly to air pollution in major cities. As a result they indicated there is need to incorporate environmental considerations into the nation's energy development and utilization strategy. Consequently, the Commission recommended, among others, reducing energy consumption by improving and expanding mass transportation and communication systems all over the country.

Zavala et al, (2009) in their work on comparison of emissions from on-road sources using a mobile laboratory under various driving and operational sampling modes stated that emissions from transportation sources, primarily on-road motor vehicles, are generally the largest contributors to criteria air pollutants such as CO, NOx, and selected volatile organic compounds (VOCs) in urban areas. They concluded that, mobile sources produce a significant fraction of the total anthropogenic emissions burden in large cities and have harmful effects on air quality at multiple spatial scales. Abam and Unachukwu (2009), in course of their work on Vehicular Emission and Air Quality Standards in Nigeria reported that in Nigeria much attention is given on general industrial pollution and pollution in oil industries, with little reference on damage of pollution caused by mobile transportation sources of air pollution, (Faboye, 1997; Iyoha, 2000; Magbabeola, 2001). Studies conducted in Kaduna and Abuja cities show higher values of CO2 concentration in heavily congested areas: $1840 \mathrm{ppm}$ for Sambo Kaduna, 1780ppm for Stadium round-about, Kaduna, and 1530ppm for A.Y.A. Abuja, 1160ppm for Asokoro Abuja, (Akpan and Ndoke; 1999). Similar work by Jimo and Ndoke (2000) at Minna, a city in Nigeria shows the maximum value of 5,000ppm for CO2 in congested area, which is still lower than WHO stipulated maximum value of 20,000ppm. The maximum value for CO emission obtained was 15ppm still lower than the base line of 48ppm stipulated by WHO and 20ppm stipulated by Federal Environmental Protection Agency of Nigeria (FEPA). The reason for this low emission concentration in Minna is due to low traffic and industrial activities in the city.

All previous works reviewed are in unison in acknowledging the high contribution of vehicle emission to air pollution. While the author's opinion on mitigation measures depended on age and research discoveries available, majority of them agree to the fact that necessary measures based on source curtailment remain the most feasible, viable and economical. This is of the course in line with the current trend in environmental management which emphasizes reduction in waste generation. Hence the focus of this paper will be on efforts at reducing air pollutants generation due to road transport in Owerri. 


\section{Method of Assessment}

The mobility needs of commuters result in the use of vehicles and the powering of the vehicles produces air pollutants, therefore, the adopted assessment and control strategy of transport - induced pollution included;

- Determination of the transport need and

- Measurement of pollution level (pollutants of interest) of the study area - Owerri Municipal, resulting from the needed vehicles for the movement.

The assessment was carried out at five strategic representative locations in the Owerri Municipal viz; Imo State University (IMSU) Junction, Amakohia Junction, Assumpta Junction, Emmanuel College Junction and MCC/Wethedral Road Junction.

The selected locations for the survey are points with high traffic and business activities. The result of the assessment was imputed into a model in terms of vehicle types and exhaust emissions, and optimized for emission reduction.

The vehicles, (in their various classes), traversing the five locations in the project area was counted over a 12-hour period - 7am to $6 \mathrm{p} . \mathrm{m}$. Based on an observed percentage occupancy of the passenger vehicles, the total passenger transportation needs at the five locations was determined. These locations record high traffic volumes within the hours of 7.30 - 9.30am (when offices and commercial activities commence) and $4.00-7.00 \mathrm{pm}$ in the evening at the close of work and market activities. The time-segmented transportation needs/loads as well as the cumulative transportation needs/loads at the locations was evaluated.

The target air pollutants - carbon monoxide (CO), Nitrogen dioxide (NO2), Hydrocarbons-Methane (CH4), were measured using standard equipment called Aeroqual/Crowcon Gasman Monitors. The measurements were at three intervals within 12 hours in a day for a total of two days at each location.

\section{Results}

\subsection{Field Survey Results}

The vehicle counts from the four approaches for each junction and survey day are collated and the cumulative figures as well as the corresponding measured pollutants are presented in tables.

\subsection{IMSU Junction}

Table 1 is for day 1 while table 2 is for day 2 .

Table 1 Cumulative vehicle types for day 1

\begin{tabular}{|c|c|c|c|c|c|c|c|c|c|}
\hline \multirow[t]{2}{*}{ Period } & \multicolumn{6}{|c|}{ Cumulative Vehicle Types } & \multicolumn{3}{|c|}{ Pollutants } \\
\hline & $\begin{array}{l}\text { Sal. } \\
\text { Veh. V1 }\end{array}$ & $\begin{array}{l}\text { Wagon } \\
\text { Veh. V2 }\end{array}$ & $\begin{array}{l}\text { Mini } \\
\text { Buses V }\end{array}$ & $\begin{array}{l}\text { Coaster } \\
\text { Buses V4 }\end{array}$ & $\begin{array}{l}\text { Big } \\
\text { Buses V }\end{array}$ & $\begin{array}{l}\text { Trucks } \\
V_{6}\end{array}$ & $\begin{array}{l}\text { CO } \\
\text { ppm }\end{array}$ & $\begin{array}{l}\mathrm{NO}_{2} \\
\mathrm{Ppm} \\
\end{array}$ & $\begin{array}{l}\mathrm{CH}_{4} \\
\mathrm{Ppm} \\
\end{array}$ \\
\hline $\begin{array}{l}\text { Morning } \\
\text { (At 7am) }\end{array}$ & 847 & 833 & 186 & 37 & 22 & 262 & 09 & 18 & 0.031 \\
\hline $\begin{array}{l}\text { Afternoon } \\
\text { (At 2pm) }\end{array}$ & 10,112 & 8261 & 2628 & 125 & 54 & 684 & 05 & 0.169 & ND \\
\hline $\begin{array}{l}\text { Evening } \\
\text { (At 6pm) }\end{array}$ & 14,579 & 12,487 & 4045 & 201 & 79 & 944 & 05 & 0.090 & ND \\
\hline & \multicolumn{6}{|c|}{ Standards } & 10 & $0.04-0.06$ & NS \\
\hline
\end{tabular}

ND: NONE DETECTED; NS: NOT STATED 
Table 2 Cumulative vehicle types for day 2

\begin{tabular}{|c|c|c|c|c|c|c|c|c|c|}
\hline \multirow[t]{2}{*}{ Period } & \multicolumn{6}{|c|}{ Cumulative Vehicle Types } & \multicolumn{3}{|c|}{ Pollutants } \\
\hline & $\begin{array}{c}\text { Sal. } \\
\text { Veh. } V_{1}\end{array}$ & $\begin{array}{l}\text { Wagon } \\
\text { Veh. } V_{2}\end{array}$ & $\begin{array}{c}\text { Mini } \\
\text { Buses } V_{3}\end{array}$ & $\begin{array}{c}\text { Coaster } \\
\text { Buses V }{ }_{4}\end{array}$ & $\begin{array}{c}\text { Big } \\
\text { Buses } V_{5}\end{array}$ & $\begin{array}{c}\text { Trucks } \\
V_{6}\end{array}$ & $\begin{array}{c}\text { CO } \\
\text { ppm }\end{array}$ & $\begin{array}{l}\mathrm{NO}_{2} \\
\mathrm{Ppm}\end{array}$ & $\begin{array}{l}\mathrm{CH}_{4} \\
\mathrm{Ppm}\end{array}$ \\
\hline $\begin{array}{l}\text { Morning } \\
\text { (At 7am) }\end{array}$ & 687 & 769 & 112 & 09 & 04 & 34 & 09 & 0.084 & ND \\
\hline $\begin{array}{l}\text { Afternoon } \\
\text { (At 2pm) }\end{array}$ & 15090 & 12661 & 2343 & 229 & 78 & 297 & 06 & 0.040 & ND \\
\hline Evening & 25022 & 20813 & 3443 & 357 & 106 & 555 & 14 & 0.086 & ND \\
\hline & \multicolumn{6}{|c|}{ Standards } & 10 & $0.04-0.06$ & NS \\
\hline
\end{tabular}

ND: NONE DETECTED; NS: NOT STATED

At this junction one of the measured values of $\mathrm{CO}$ is above the standard, two values are close to the standard while three are below the standard. Five of the Nitrogen dioxide values are above the standard while one is within the standard. Methane was only detected on one out of the six times.

\subsection{Amakohia Junction}

The corresponding figures for Amakohia Junction are presented in Tables 3 and 4.

Table 3 Cumulative vehicle types for day 1

\begin{tabular}{|c|c|c|c|c|c|c|c|c|c|}
\hline \multirow[t]{2}{*}{ Period } & \multicolumn{6}{|c|}{ Cumulative Vehicle Types } & \multicolumn{3}{|c|}{ Pollutants } \\
\hline & $\begin{array}{c}\text { Sal. Veh. } \\
V_{1}\end{array}$ & $\begin{array}{l}\text { Wagon } \\
\text { Veh. } V_{2}\end{array}$ & $\begin{array}{c}\text { Mini } \\
\text { Buses } V_{3}\end{array}$ & $\begin{array}{c}\text { Coaster } \\
\text { Buses V } 4\end{array}$ & $\begin{array}{c}\text { Big } \\
\text { Buses } V_{5}\end{array}$ & $\begin{array}{c}\text { Trucks } \\
V_{6}\end{array}$ & $\begin{array}{c}\text { CO } \\
\text { ppm }\end{array}$ & $\begin{array}{l}\mathrm{NO}_{2} \\
\mathrm{Ppm}\end{array}$ & $\begin{array}{l}\mathrm{CH}_{4} \\
\mathrm{Ppm}\end{array}$ \\
\hline $\begin{array}{l}\text { Morning } \\
\text { (At 7am) }\end{array}$ & 754 & 597 & 160 & 08 & 05 & 57 & 3 & 0.134 & ND \\
\hline $\begin{array}{l}\text { Afternoon } \\
\text { (At 2pm) }\end{array}$ & 7628 & 7110 & 1435 & 121 & 70 & 402 & 3 & 0.073 & ND \\
\hline $\begin{array}{l}\text { Evening } \\
\text { (At 6pm) }\end{array}$ & 14057 & 13831 & 3285 & 297 & 127 & 783 & 7 & 0.111 & ND \\
\hline & \multicolumn{6}{|l|}{ Standards } & 10 & $0.04-0.06$ & NS \\
\hline
\end{tabular}

Table 4 Cumulative vehicle types for day 2

\begin{tabular}{|c|c|c|c|c|c|c|c|c|c|}
\hline \multirow[t]{2}{*}{ Period } & \multicolumn{6}{|c|}{ Cumulative Vehicle Types } & \multicolumn{3}{|c|}{ Pollutants } \\
\hline & $\begin{array}{l}\text { Sal. Veh. } \\
\text { V }_{1}\end{array}$ & $\begin{array}{l}\text { Wagon } \\
\text { Veh. } V_{2}\end{array}$ & $\begin{array}{l}\text { Mini } \\
\text { Buses V }\end{array}$ & $\begin{array}{l}\text { Coaster } \\
\text { Buses V } 4\end{array}$ & $\begin{array}{l}\text { Big } \\
\text { Buses V }\end{array}$ & $\begin{array}{l}\text { Trucks } \\
\mathrm{V}_{6}\end{array}$ & $\begin{array}{l}\text { CO } \\
\text { ppm }\end{array}$ & $\begin{array}{l}\mathrm{NO}_{2} \\
\mathrm{Ppm}\end{array}$ & $\begin{array}{l}\mathrm{CH}_{4} \\
\mathrm{Ppm}\end{array}$ \\
\hline $\begin{array}{l}\text { Morning } \\
\text { (At 7am) }\end{array}$ & 607 & 601 & 63 & 05 & 02 & 42 & 7 & 0.06 & ND \\
\hline $\begin{array}{l}\text { Afternoon } \\
\text { (At 2pm) }\end{array}$ & 10581 & 8187 & 3405 & 544 & 62 & 435 & 6 & 0.043 & ND \\
\hline $\begin{array}{l}\text { Evening } \\
\text { (At 6pm) }\end{array}$ & 16218 & 15311 & 5526 & 645 & 120 & 806 & 8 & 0.073 & ND \\
\hline & \multicolumn{6}{|l|}{ Standards } & 10 & $0.04-0.06$ & NS \\
\hline
\end{tabular}


Three of the Carbon monoxide values at this junction are well below the standard while three are very close to it. Four of the nitrogen dioxide values are well above the standard while two are within the standard. Methane was not detected.

\subsection{Assumpta Junction}

The corresponding figures for Assumpta Junction are presented in tables 5 and 6.

Table 5 Cumulative vehicle types for day 1

\begin{tabular}{|c|c|c|c|c|c|c|c|c|c|}
\hline \multirow[t]{2}{*}{ Period } & \multicolumn{6}{|c|}{ Cumulative Vehicle Types } & \multicolumn{3}{|c|}{ Pollutants } \\
\hline & $\begin{array}{c}\text { Sal. } \\
\text { Veh. V1 }\end{array}$ & $\begin{array}{l}\text { Wagon } \\
\text { Veh. V } 2\end{array}$ & $\begin{array}{c}\text { Mini } \\
\text { Buses V }\end{array}$ & $\begin{array}{c}\text { Coaster } \\
\text { Buses V } 4\end{array}$ & $\begin{array}{c}\text { Big Buses } \\
V_{5}\end{array}$ & $\begin{array}{c}\text { Trucks } \\
\mathrm{V}_{6}\end{array}$ & $\begin{array}{c}\text { CO } \\
\text { Ppm }\end{array}$ & $\begin{array}{l}\mathrm{NO}_{2} \\
\mathrm{Ppm}\end{array}$ & $\begin{array}{l}\mathrm{CH}_{4} \\
\mathrm{Ppm}\end{array}$ \\
\hline $\begin{array}{l}\text { Morning } \\
\text { (At 7am) }\end{array}$ & 743 & 874 & 215 & 13 & 15 & 69 & ND & 0.090 & 1.00 \\
\hline $\begin{array}{l}\text { Afternoon } \\
\text { (At 2pm) }\end{array}$ & 11332 & 10304 & 2152 & 135 & 74 & 714 & 6.00 & 0.060 & ND \\
\hline $\begin{array}{l}\text { Evening (At } \\
6 \mathrm{pm})\end{array}$ & 20429 & 16775 & 3637 & 215 & 119 & 1727 & 17.00 & 0.005 & ND \\
\hline & \multicolumn{6}{|c|}{ Standards } & 10 & $0.04-0.06$ & NS \\
\hline
\end{tabular}

Table 6 Cumulative vehicle types for day 2

\begin{tabular}{|c|c|c|c|c|c|c|c|c|c|}
\hline \multirow[t]{2}{*}{ Period } & \multicolumn{6}{|c|}{ Cumulative Vehicle Types } & \multicolumn{3}{|c|}{ Pollutants } \\
\hline & $\begin{array}{l}\text { Sal. } \\
\text { Veh. V1 }\end{array}$ & $\begin{array}{l}\text { Wagon } \\
\text { Veh. V2 }\end{array}$ & $\begin{array}{l}\text { Mini } \\
\text { Buses } V_{3}\end{array}$ & $\begin{array}{l}\text { Coaster } \\
\text { Buses V4 }\end{array}$ & $\begin{array}{l}\text { Big } \\
\text { Buses V5 }\end{array}$ & $\begin{array}{l}\text { Trucks } \\
\mathrm{V}_{6}\end{array}$ & $\begin{array}{l}\text { CO } \\
\text { Ppm }\end{array}$ & $\begin{array}{l}\mathrm{NO}_{2} \\
\mathrm{Ppm}\end{array}$ & $\begin{array}{l}\mathrm{CH}_{4} \\
\text { Ppm }\end{array}$ \\
\hline $\begin{array}{l}\text { Morning } \\
\text { (At 7am) }\end{array}$ & 715 & 586 & 229 & 15 & 22 & 75 & 3.00 & 0.086 & ND \\
\hline $\begin{array}{l}\text { Afternoon } \\
\text { (At 2pm) }\end{array}$ & 9733 & 11291 & 2908 & 275 & 234 & 507 & 11.00 & 087 & ND \\
\hline Evening & 14534 & 18853 & 3819 & 382 & 357 & 835 & 3.00 & 0.137 & ND \\
\hline & \multicolumn{6}{|c|}{ Standards } & 10 & 0.04-0.06 & NS \\
\hline
\end{tabular}

Two of the Carbon monoxide values recorded at this junction are above the standard while four values are below the standard. Four values of nitrogen-dioxide are above standards while two are within the standards. Only on one occasion was a value recorded for methane.

\subsection{Emmanuel College}

The corresponding figures for Emmanuel College junction are presented in Tables 4.7 and 4.8. 
Table 7 Cumulative vehicle types for day 1

\begin{tabular}{|c|c|c|c|c|c|c|c|c|c|}
\hline \multirow[t]{2}{*}{ Period } & \multicolumn{6}{|c|}{ Cumulative Vehicle Types } & \multicolumn{3}{|c|}{ Pollutants } \\
\hline & $\begin{array}{c}\text { Sal. } \\
\text { Veh. } V_{1}\end{array}$ & $\begin{array}{l}\text { Wagon } \\
\text { Veh. } V_{2}\end{array}$ & $\begin{array}{c}\text { Mini } \\
\text { Buses } V_{3}\end{array}$ & $\begin{array}{c}\text { Coaster } \\
\text { Buses } V_{4}\end{array}$ & $\begin{array}{c}\text { Big } \\
\text { Buses } V_{5}\end{array}$ & $\begin{array}{c}\text { Trucks } \\
V_{6}\end{array}$ & $\begin{array}{c}\text { CO } \\
\text { Ppm }\end{array}$ & $\begin{array}{l}\mathrm{NO}_{2} \\
\mathrm{Ppm}\end{array}$ & $\begin{array}{l}\mathrm{CH}_{4} \\
\mathrm{Ppm}\end{array}$ \\
\hline $\begin{array}{l}\text { Morning } \\
\text { (At 7am) }\end{array}$ & 1498 & 2816 & 527 & 89 & 60 & 158 & 31.00 & 0.070 & ND \\
\hline $\begin{array}{l}\text { Afternoon } \\
\text { (At 2pm) }\end{array}$ & 11154 & 11635 & 5014 & 473 & 316 & 584 & 17.00 & 0.048 & ND \\
\hline \multirow{2}{*}{$\begin{array}{l}\text { Evening } \\
\text { (At 6pm) }\end{array}$} & 21184 & 20391 & 11921 & 953 & 607 & 1276 & 7.00 & 0.068 & 1.00 \\
\hline & \multicolumn{6}{|c|}{ Standards } & 10 & $0.04-0.06$ & NS \\
\hline
\end{tabular}

Table 8 Cumulative vehicle types for day 2

\begin{tabular}{|c|c|c|c|c|c|c|c|c|c|}
\hline \multirow[t]{2}{*}{ Period } & \multicolumn{6}{|c|}{ Cumulative Vehicle Types } & \multicolumn{3}{|c|}{ Pollutants } \\
\hline & $\begin{array}{c}\text { Sal. } \\
\text { Veh. } V_{1}\end{array}$ & $\begin{array}{l}\text { Wagon } \\
\text { Veh. } V_{2}\end{array}$ & $\begin{array}{c}\text { Mini } \\
\text { Buses } V_{3}\end{array}$ & $\begin{array}{c}\text { Coaster } \\
\text { Buses V V }\end{array}$ & $\begin{array}{c}\text { Big } \\
\text { Buses } V_{5}\end{array}$ & $\begin{array}{c}\text { Trucks } \\
\mathrm{V}_{6}\end{array}$ & $\begin{array}{c}\text { CO } \\
\text { Ppm }\end{array}$ & $\begin{array}{c}\mathrm{NO}_{2} \\
\mathrm{Ppm}\end{array}$ & $\begin{array}{c}\mathrm{CH}_{4} \\
\mathrm{Ppm}\end{array}$ \\
\hline $\begin{array}{l}\text { Morning } \\
\text { (At 7am) }\end{array}$ & 876 & 2135 & 434 & 38 & 33 & 180 & 80.00 & 0.073 & 1.00 \\
\hline $\begin{array}{l}\text { Afternoon } \\
\text { (At 2pm) }\end{array}$ & 27734 & 32567 & 6706 & 114 & 128 & 1318 & 22.00 & 0.092 & ND \\
\hline $\begin{array}{l}\text { Evening } \\
\text { (At 6pm) }\end{array}$ & 34101 & 38368 & 9496 & 228 & 227 & 1697 & 13.00 & 0.182 & ND \\
\hline & \multicolumn{6}{|c|}{ Standards } & 10 & $0.04-0.06$ & NS \\
\hline
\end{tabular}

All the values of the Carbon-monoxide measured here are above the standard and in most cases about double the standard. All but one nitrogen dioxide values are above the standard while methane was detected on two out of the six times.

\subsection{Wethedral/MCC Junction}

The corresponding figures for the Wethedral/MCC junction are presented in Tables 9 and 10.

Table 9 Cumulative vehicle types for day 1

\begin{tabular}{|c|c|c|c|c|c|c|c|c|c|}
\hline \multirow[t]{2}{*}{ Period } & \multicolumn{6}{|c|}{ Cumulative Vehicle Types } & \multicolumn{3}{|c|}{ Pollutants } \\
\hline & $\begin{array}{c}\text { Sal. } \\
\text { Veh. } \text { V }_{1}\end{array}$ & $\begin{array}{l}\text { Wagon } \\
\text { Veh. } V_{2}\end{array}$ & $\begin{array}{c}\text { Mini } \\
\text { Buses } V_{3}\end{array}$ & $\begin{array}{c}\text { Coaster } \\
\text { Buses V V }\end{array}$ & $\begin{array}{c}\text { Big } \\
\text { Buses V }\end{array}$ & $\begin{array}{c}\text { Trucks } \\
\mathbf{V}_{6}\end{array}$ & $\begin{array}{c}\text { CO } \\
\text { Ppm }\end{array}$ & $\begin{array}{l}\mathrm{NO}_{2} \\
\mathrm{Ppm}\end{array}$ & $\begin{array}{l}\mathrm{CH}_{4} \\
\mathrm{Ppm}\end{array}$ \\
\hline $\begin{array}{l}\text { Morning } \\
\text { (At 7am) }\end{array}$ & 1108 & 2353 & 112 & 18 & 24 & 86 & 20.00 & 0.072 & ND \\
\hline $\begin{array}{l}\text { Afternoon } \\
\text { (At 2pm) }\end{array}$ & 16151 & 15702 & 389 & 62 & 85 & 316 & 13.00 & 0.084 & ND \\
\hline $\begin{array}{l}\text { Evening } \\
\text { (At 6pm) }\end{array}$ & 30647 & 23098 & 808 & 164 & 195 & 633 & 21.00 & 0.126 & ND \\
\hline & \multicolumn{6}{|c|}{ Standards } & 10 & $0.04-0.06$ & NS \\
\hline
\end{tabular}


Table 10 Cumulative vehicle types for day 2

\begin{tabular}{|c|c|c|c|c|c|c|c|c|c|}
\hline \multirow[t]{2}{*}{ Period } & \multicolumn{6}{|c|}{ Cumulative Vehicle Types } & \multicolumn{3}{|c|}{ Pollutants } \\
\hline & $\begin{array}{l}\text { Sal. } \\
\text { Veh. } V_{1}\end{array}$ & $\begin{array}{l}\text { Wagon } \\
\text { Veh. } V_{2}\end{array}$ & $\begin{array}{l}\text { Mini } \\
\text { Buses } V_{3}\end{array}$ & $\begin{array}{l}\text { Coaster } \\
\text { Buses V V }\end{array}$ & $\begin{array}{l}\text { Big } \\
\text { Buses V }\end{array}$ & $\begin{array}{l}\text { Trucks } \\
V_{6}\end{array}$ & $\begin{array}{l}\text { CO } \\
\text { Ppm }\end{array}$ & $\begin{array}{l}\mathrm{NO}_{2} \\
\mathrm{Ppm}\end{array}$ & $\begin{array}{l}\mathrm{CH}_{4} \\
\mathrm{Ppm}\end{array}$ \\
\hline $\begin{array}{l}\text { Morning } \\
\text { (At 7am) }\end{array}$ & 383 & 734 & 45 & 04 & 06 & 35 & 22.00 & 0.075 & ND \\
\hline $\begin{array}{l}\text { Afternoon } \\
\text { (At 2pm) }\end{array}$ & 6639 & 13537 & 579 & 61 & 76 & 254 & 17.00 & ND & ND \\
\hline $\begin{array}{l}\text { Evening } \\
\text { (At 6pm) }\end{array}$ & 10509 & 19579 & 883 & 106 & 107 & 405 & 3.00 & 0.091 & ND \\
\hline & \multicolumn{6}{|c|}{ Standards } & 10 & $0.04-0.06$ & NS \\
\hline
\end{tabular}

ND: NONE DETECTED; NS: NOT STATED

At this junction all but one value of Carbon monoxide is well above the standard. On five of the six times the nitrogen dioxide values are above the standard while methane was not detected.

\subsection{Exhaust Emission of vehicles}

The representative vehicles exhaust emissions for the three pollutants of interest are presented in Table 11, as follows;

Table 11 Exhaust Emission of Different Vehicles

\begin{tabular}{|l|c|c|c|}
\hline \multirow{2}{*}{ Vehicle } & \multicolumn{3}{|c|}{ Pollutants } \\
\cline { 2 - 4 } & $\begin{array}{c}\mathrm{CO}, \\
\mathrm{ppm}\end{array}$ & $\begin{array}{c}\mathrm{NO}_{2}, \\
\mathrm{ppm}\end{array}$ & $\begin{array}{c}\mathrm{CH}_{4}, \\
\mathrm{ppm}\end{array}$ \\
\hline Car 1 & 234.30 & 0.56 & 284.00 \\
\hline Car 2 & 234.30 & 0.30 & 5613.00 \\
\hline Car 3 & 234.30 & 0.60 & 4227.00 \\
\hline Average & 234.30 & 0.49 & 4229.00 \\
\hline & & & \\
\hline Wagon 1 & 173.80 & 0.93 & 79.00 \\
\hline Wagon 2 & 68.80 & 0.49 & 87.00 \\
\hline Wagon 3 & 234.30 & 0.04 & 79.00 \\
\hline Average & 158.97 & 0.49 & 81.67 \\
\hline & & & \\
\hline Mini Bus 1 & 234.30 & 0.28 & 3797.00 \\
\hline Mini Bus 2 & 234.30 & 0.22 & 4461.00 \\
\hline Mini Bus 3 & 209.20 & 0.15 & 512.00 \\
\hline Average & 225.93 & 0.22 & 2923.33 \\
\hline & & & \\
\hline Coaster Bus 1 & 234.30 & 0.63 & 479.00 \\
\hline Coaster Bus 2 & 234.30 & 0.63 & 444.00 \\
\hline
\end{tabular}




\begin{tabular}{|l|c|c|c|}
\hline Coaster Bus 3 & 234.30 & 0.63 & 396.00 \\
\hline Average & 234.30 & 0.63 & 439.67 \\
\hline & & & \\
\hline Big Bus 1 & ND & 5.37 & 14.00 \\
\hline Big Bus 2 & ND & 5.37 & 14.00 \\
\hline Big Bus 3 & ND & 5.37 & 10.00 \\
\hline Average & ND & 5.37 & 12.67 \\
\hline & & & \\
\hline Truck 1 & 254.60 & 1.03 & 18.00 \\
\hline Truck 2 & 246.80 & 1.26 & 16.00 \\
\hline Truck 3 & 254.60 & 1.27 & 20.00 \\
\hline Average & 252.00 & 1.19 & 18.00 \\
\hline
\end{tabular}

ND: NONE DETECTED; NS: NOT STATED

\subsection{Analysis}

Applying the principle of worst-case scenario on the collated cumulative results, including the measured pollutants for the survey locations yields the critical values in Tables 12,13,14,15, and 16.

Table 12 Cumulative result for IMSU junction

\begin{tabular}{|c|c|c|c|c|c|c|c|c|c|}
\hline \multirow[t]{2}{*}{ Period } & \multicolumn{6}{|c|}{ Cumulative Vehicle Types } & \multicolumn{3}{|c|}{ Pollutants } \\
\hline & $\begin{array}{l}\text { Sal. Veh. } \\
\text { V }_{1}\end{array}$ & $\begin{array}{l}\text { Wagon } \\
\text { Veh. } V_{2}\end{array}$ & $\begin{array}{l}\text { Mini } \\
\text { Buses V }\end{array}$ & $\begin{array}{l}\text { Coaster } \\
\text { Buses V } 4\end{array}$ & $\begin{array}{l}\text { Big } \\
\text { Buses V } 5\end{array}$ & $\begin{array}{l}\text { Trucks } \\
V_{6}\end{array}$ & $\begin{array}{l}\text { CO } \\
\text { Ppm }\end{array}$ & $\begin{array}{l}\mathrm{NO}_{2} \\
\mathrm{ppm}\end{array}$ & $\begin{array}{l}\mathrm{CH}_{4} \\
\mathrm{Ppm}\end{array}$ \\
\hline $\begin{array}{l}\text { Morning } \\
\text { (At 7am) }\end{array}$ & 847 & 833 & 186 & 37 & 22 & 262 & 09 & 18 & 0.031 \\
\hline $\begin{array}{l}\text { Afternoon } \\
\text { (At 2pm) }\end{array}$ & 15090 & 12661 & 3403 & 229 & 78 & 684 & 06 & 0.169 & ND \\
\hline $\begin{array}{l}\text { Evening } \\
\text { (At 6pm) }\end{array}$ & 25022 & 20813 & 4820 & 357 & 106 & 944 & 14 & 0.090 & ND \\
\hline
\end{tabular}

Table 13 Cumulative result for amakohia junction

\begin{tabular}{|c|c|c|c|c|c|c|c|c|c|}
\hline \multirow[t]{2}{*}{ Period } & \multicolumn{6}{|c|}{ Cumulative Vehicle Types } & \multicolumn{3}{|c|}{ Pollutants } \\
\hline & $\begin{array}{c}\text { Sal. Veh. } \\
\mathbf{V}_{\mathbf{1}}\end{array}$ & $\begin{array}{l}\text { Wagon } \\
\text { Veh. } V_{2}\end{array}$ & $\begin{array}{c}\text { Mini } \\
\text { Buses } V_{3}\end{array}$ & $\begin{array}{c}\text { Coaster } \\
\text { Buses } V_{4}\end{array}$ & $\begin{array}{c}\text { Big } \\
\text { Buses } V_{5}\end{array}$ & $\begin{array}{c}\text { Trucks } \\
V_{6}\end{array}$ & $\begin{array}{c}\text { CO } \\
\text { Ppm }\end{array}$ & $\begin{array}{l}\mathrm{NO}_{2} \\
\text { ppm }\end{array}$ & $\begin{array}{l}\mathrm{CH}_{4} \\
\mathrm{Ppm}\end{array}$ \\
\hline $\begin{array}{l}\text { Morning } \\
\text { (At 7am) }\end{array}$ & 754 & 601 & 160 & 08 & 05 & 57 & 7 & 0.134 & ND \\
\hline $\begin{array}{l}\text { Afternoon } \\
\text { (At 2pm) }\end{array}$ & 10581 & 8187 & 3405 & 544 & 70 & 435 & 6 & 0.073 & ND \\
\hline $\begin{array}{l}\text { Evening } \\
\text { (At 6pm) }\end{array}$ & 16218 & 15311 & 5526 & 645 & 127 & 806 & 8 & 0.111 & ND \\
\hline
\end{tabular}


Table 14 Cumulative result for asumpta junction

\begin{tabular}{|c|c|c|c|c|c|c|c|c|c|}
\hline \multirow[t]{2}{*}{ Period } & \multicolumn{6}{|c|}{ Cumulative Vehicle Types } & \multicolumn{3}{|c|}{ Pollutants } \\
\hline & $\begin{array}{l}\text { Sal. Veh. } \\
\text { V }_{1}\end{array}$ & $\begin{array}{l}\text { Wagon } \\
\text { Veh. } V_{2}\end{array}$ & $\begin{array}{l}\text { Mini } \\
\text { Buses V }\end{array}$ & $\begin{array}{l}\text { Coaster } \\
\text { Buses V } 4\end{array}$ & $\begin{array}{l}\text { Big } \\
\text { Buses } V_{5}\end{array}$ & $\begin{array}{l}\text { Trucks } \\
V_{6}\end{array}$ & $\begin{array}{l}\text { CO } \\
\text { Ppm }\end{array}$ & $\begin{array}{l}\mathrm{NO}_{2} \\
\mathrm{ppm}\end{array}$ & $\begin{array}{l}\mathrm{CH}_{4} \\
\mathrm{Ppm}\end{array}$ \\
\hline $\begin{array}{l}\text { Morning } \\
\text { (At 7am) }\end{array}$ & 743 & 874 & 229 & 15 & 22 & 75 & 3.00 & 0.090 & 1.00 \\
\hline $\begin{array}{l}\text { Afternoon } \\
\text { (At 2pm) }\end{array}$ & 11332 & 11291 & 2908 & 275 & 234 & 714 & 11.00 & 0.087 & ND \\
\hline $\begin{array}{l}\text { Evening } \\
\text { (At 6pm) }\end{array}$ & 20429 & 18853 & 3819 & 382 & 357 & 1727 & 17.00 & 0.137 & ND \\
\hline
\end{tabular}

Table 15 Cumulative result for emmanuel college junction

\begin{tabular}{|c|c|c|c|c|c|c|c|c|c|}
\hline \multirow[t]{2}{*}{ Period } & \multicolumn{6}{|c|}{ Cumulative Vehicle Types } & \multicolumn{3}{|c|}{ Pollutants } \\
\hline & $\begin{array}{c}\text { Sal. Veh. } \\
\mathrm{V}_{1}\end{array}$ & $\begin{array}{l}\text { Wagon } \\
\text { Veh. } V_{2}\end{array}$ & $\begin{array}{c}\text { Mini } \\
\text { Buses } V_{3}\end{array}$ & $\begin{array}{c}\text { Coaster } \\
\text { Buses } V_{4}\end{array}$ & $\begin{array}{c}\text { Big } \\
\text { Buses } V_{5}\end{array}$ & $\begin{array}{c}\text { Trucks } \\
\mathrm{V}_{6}\end{array}$ & $\begin{array}{c}\text { CO } \\
\text { Ppm }\end{array}$ & $\begin{array}{l}\mathrm{NO}_{2} \\
\mathrm{Ppm}\end{array}$ & $\begin{array}{l}\mathrm{CH}_{4} \\
\text { Ppm }\end{array}$ \\
\hline $\begin{array}{l}\text { Morning } \\
\text { (At 7am) }\end{array}$ & 1498 & 2816 & 527 & 89 & 60 & 180 & 80.00 & 0.073 & 1.00 \\
\hline $\begin{array}{l}\text { Afternoon } \\
\text { (At 2pm) }\end{array}$ & 11154 & 11635 & 6706 & 473 & 316 & 584 & 22.00 & 0.092 & ND \\
\hline $\begin{array}{l}\text { Evening (At } \\
6 \mathrm{pm} \text { ) }\end{array}$ & 34101 & 38368 & 11921 & 953 & 607 & 1697 & 13.00 & 0.182 & 1.00 \\
\hline
\end{tabular}

Table 16 Cumulative result for wethedral/MCC junction

\begin{tabular}{|c|c|c|c|c|c|c|c|c|c|}
\hline \multirow[t]{2}{*}{ Period } & \multicolumn{6}{|c|}{ Cumulative Vehicle Types } & \multicolumn{3}{|c|}{ Pollutants } \\
\hline & $\begin{array}{c}\text { Sal. Veh. } \\
\text { V }_{1}\end{array}$ & $\begin{array}{l}\text { Wagon } \\
\text { Veh. } V_{2}\end{array}$ & $\begin{array}{c}\text { Mini } \\
\text { Buses } V_{3}\end{array}$ & $\begin{array}{c}\text { Coaster } \\
\text { Buses V } 4\end{array}$ & $\begin{array}{c}\text { Big } \\
\text { Buses } V_{5}\end{array}$ & $\begin{array}{c}\text { Trucks } \\
V_{6}\end{array}$ & $\begin{array}{c}\text { CO } \\
\text { Ppm }\end{array}$ & $\begin{array}{l}\mathrm{NO}_{2} \\
\mathrm{Ppm}\end{array}$ & $\begin{array}{l}\mathrm{CH}_{4} \\
\mathrm{Ppm}\end{array}$ \\
\hline $\begin{array}{l}\text { Morning } \\
\text { (At 7am) }\end{array}$ & 1108 & 2353 & 112 & 18 & 24 & 86 & 22.00 & 0.075 & ND \\
\hline $\begin{array}{l}\text { Afternoon } \\
\text { (At 2pm) }\end{array}$ & 16151 & 15702 & 379 & 62 & 85 & 316 & 17.00 & 0.084 & ND \\
\hline $\begin{array}{l}\text { Evening } \\
\text { (At 6pm) }\end{array}$ & 30647 & 23098 & 883 & 164 & 195 & 633 & 21.00 & 0.126 & ND \\
\hline
\end{tabular}

\subsection{Commuters Traversing the Junctions}

The total commuters traversing the survey locations is the product of the number of vehicles and their weighted carrying capacities. From field observations the vehicles were averagely $60 \%$ loaded such that the following weighted capacities are derived. 
Table 17 Passenger carrying capacity of vehicles

\begin{tabular}{|l|c|c|}
\hline Vehicle Type & Design Capacity & Weighted Capacity \\
\hline Saloon Vehicles & 5 & 3 \\
\hline Wagon Vehicles & 8 & 5 \\
\hline Mini Buses & 15 & 9 \\
\hline Coaster Buses & 33 & 20 \\
\hline Big Buses & 53 & 32 \\
\hline
\end{tabular}

Using the weighted carrying capacities and the cumulative vehicles accessing the locations, the total commuters traversing the locations are calculated as shown in Tables 18, 19, 20, 21 and 22 respectively.

Table 18 Total Commuters Traversing IMSU Junction in a 12-Hour Day

\begin{tabular}{|l|c|c|c|}
\hline Veh. Types & Cumulative No & Weighted Capacity & Total Commuters \\
\hline Saloon Veh. $V_{1}$ & 25022 & 3 & 75,066 \\
\hline Wagon Veh. $V_{2}$ & 20813 & 5 & 104,065 \\
\hline Mini Buses $V_{3}$ & 4820 & 9 & 43,380 \\
\hline Coaster Buses $V_{4}$ & 357 & 20 & 7,140 \\
\hline Big Buses $V_{5}$ & 106 & 32 & 3,392 \\
\hline \multicolumn{3}{|c|}{ Total 51,118} & 233043 \\
\hline
\end{tabular}

Table 19 Total Commuters Traversing Amakohia Junction in a 12-Hour Day

\begin{tabular}{|l|c|c|c|}
\hline Veh. Types & Cumulative No & Weighted Capacity & Total Commuters \\
\hline Saloon Veh. $V_{1}$ & 1621 & 3 & 4863 \\
\hline Wagon Veh. $V_{2}$ & 1531 & 5 & 7655 \\
\hline Mini Buses $V_{3}$ & 5526 & 9 & 49734 \\
\hline Coaster Buses $V_{4}$ & 645 & 20 & 12900 \\
\hline Big Buses $V_{5}$ & 127 & 32 & 4064 \\
\hline \multicolumn{3}{|c|}{ Total 9450} & 79,216 \\
\hline
\end{tabular}

Table 20 Total Commuters Traversing Assumpta Junction in a 12-Hour Day

\begin{tabular}{|l|c|c|c|}
\hline \multicolumn{1}{|c|}{ Veh. Types } & Cumulative No & Weighted Capacity & Total Commuters \\
\hline Saloon Veh. $V_{1}$ & 20429 & 3 & 61,287 \\
\hline Wagon Veh. $V_{2}$ & 18853 & 5 & 94,245 \\
\hline Mini Buses $V_{3}$ & 3819 & 9 & 34,371 \\
\hline Coaster Buses $V_{4}$ & 382 & 20 & 7,640 \\
\hline Big Buses V5 & 357 & 32 & 11,424 \\
\hline \multicolumn{3}{|c|}{ Total 43,840 } & 208,987 \\
\hline
\end{tabular}


Table 21 Total Commuters Traversing Emmanuel College Junction in a 12-Hour Day

\begin{tabular}{|l|c|c|c|}
\hline \multicolumn{1}{|c|}{ Veh. Types } & Cumulative No & Weighted Capacity & Total Commuters \\
\hline Saloon Veh. $V_{1}$ & 34101 & 3 & 102303 \\
\hline Wagon Veh. $V_{2}$ & 38368 & 5 & 191,040 \\
\hline Mini Buses $V_{3}$ & 11921 & 9 & 107,289 \\
\hline Coaster Buses $V_{4}$ & 953 & 20 & 19,060 \\
\hline Big Buses $V_{5}$ & 607 & 32 & 19,424 \\
\hline \multicolumn{3}{|c|}{ Total 85,950 } & 439,916 \\
\hline
\end{tabular}

Table 22 Total Commuters Traversing Wethedral/MCC Junction in a 12-Hour Day

\begin{tabular}{|l|c|c|c|}
\hline Veh. Types & Cumulative No & Weighted Capacity & Total Commuters \\
\hline Saloon Veh. $V_{1}$ & 30647 & 3 & 91,941 \\
\hline Wagon Veh. $V_{2}$ & 23098 & 5 & 115,490 \\
\hline Mini Buses $V_{3}$ & 883 & 9 & 7,947 \\
\hline Coaster Buses $V_{4}$ & 164 & 20 & 3,280 \\
\hline Big Buses $V_{5}$ & 195 & 32 & 6,240 \\
\hline \multicolumn{3}{|c|}{ Total 54,987} & 224,898 \\
\hline
\end{tabular}

Of the five junctions investigated Emmanuel College Junction with a total commuters number of 439,916 recorded the highest number of vehicles and commuters traversing and highest values of pollutants. Further studies will therefore be based on the results from it, as a worst case scenario.

Table 23 shows the total contribution of the three pollutants of interest into the environment by the vehicles traversing the Emmanuel college junction within 12 hours of the day.

Table 23 Total Pollutants Contribution of Vehicles that Accessed Emmanuel College Junction

\begin{tabular}{|c|c|c|c|c|c|c|c|}
\hline \multirow[t]{2}{*}{ Veh. Types } & \multirow{2}{*}{$\begin{array}{l}\text { Total } \\
\text { Veh. }\end{array}$} & \multicolumn{2}{|c|}{ Carbon Monoxide } & \multicolumn{2}{|c|}{ Nitrogen Dioxide } & \multicolumn{2}{|c|}{ Methane } \\
\hline & & Rate & $\begin{array}{c}\text { Total } \\
\text { x } 10^{3} \text { ppm }\end{array}$ & Rate & $\begin{array}{c}\text { Total } \\
\text { x } 10^{3} \text { ppm }\end{array}$ & Rate & $\begin{array}{c}\text { Total } \\
\times 10^{6} \mathrm{ppm}\end{array}$ \\
\hline Saloon Cars V 1 & 34101 & 234.3 & 7989.86 & 0.49 & 16.71 & 422.9 & 144.21 \\
\hline Wagon Veh.V 2 & 38368 & 158.97 & 6099.36 & 0.49 & 18.80 & 81.67 & 3.13 \\
\hline Mini Buses $V_{3}$ & 11921 & 225.93 & 2693.31 & 0.22 & 2.622 & 2923.33 & 34.85 \\
\hline Coaster Buses $V_{4}$ & 953 & 234.30 & 223.29 & 0.63 & 0.6 & 439.67 & 0.42 \\
\hline Big Buses $V_{5}$ & 607 & 42 & 5.49 & 5.37 & 3.26 & 12.67 & 0.0077 \\
\hline Total & 85,950 & & $\begin{array}{c}17,031.29 \mathrm{x} \\
10^{3} \mathrm{ppm}\end{array}$ & & $\begin{array}{c}41.992 \\
\times 10^{3} \mathrm{ppm}\end{array}$ & & $\begin{array}{c}182.62 \\
\times 10^{6} \mathrm{ppm}\end{array}$ \\
\hline
\end{tabular}




\subsection{Model Development}

The aim of the model is to achieve a remix of the vehicles such that the total commuters of 439,916 are served with a reduction in number of vehicles and pollutants released into the environment. This can be obtained by optimizing the model equations given in equations 1 to 5 below;

Solving the Objective function

$$
3 v_{1}+5 v_{2}+9 v_{3}+20 v_{4}+32 v_{5}=439916 \quad \text { Eqn }
$$

Subject to:

For a $40 \%$ reduction in $\mathrm{CO}$,

$$
234.3 v_{1}+158.97 v_{2}+225.93 v_{3}+234.3 v_{4}+42 v_{5}=10,218.774 \times 10^{3} \quad \text { Eqn (2) }
$$

For a $50 \%$ reduction in $\mathrm{NO}_{2}$,

$$
0.49 v_{1}+0.49 v_{2}+0.22 v_{3}+0.63 v_{4}+5.51 v_{5}=20.996 \times 10^{3}
$$

For a $50 \%$ reduction in $\mathrm{CH}_{4}$,

$$
4229 v_{1}+81.67 v_{2}+2923.33 v_{3}+439.67 v_{4}+12.67 v_{5}=91.31 \times 10^{6}
$$

That $80 \%$ of the commuters use buses

$$
0+0+9 v_{3}+20 v_{4}+32 v_{5}=351,933
$$

where $\mathrm{v}_{1}, \mathrm{~V}_{2}, \mathrm{~V}_{3}, \mathrm{~V}_{4}, \& \mathrm{~V}_{5}$ are vehicle types.

It should be noted that the ambient pollutants measured indicated that a reduction by about half of the emission will bring them within the accepted standards; tables 12 to 16.

In Matrix form the five equations are thus;

\begin{tabular}{|l|l|l|l|l|l|}
\hline $\mathbf{V}_{\mathbf{1}}$ & $\mathbf{V}_{\mathbf{2}}$ & $\mathbf{V}_{\mathbf{3}}$ & $\mathbf{V}_{\mathbf{4}}$ & $\mathbf{V}_{\mathbf{5}}$ & \\
\hline 3 & 5 & 9 & 20 & 32 & 439,916 \\
\hline 234.3 & 158.97 & 225.93 & 234.3 & 42 & $10,218,774$ \\
\hline 0.49 & 0.49 & 0.22 & 0.63 & 5.51 & 20,996 \\
\hline 4229 & 81.67 & 2923.33 & 439.67 & 12.67 & $91,310,000$ \\
\hline 0 & 0 & 9 & 20 & 32 & 351,933 \\
\hline
\end{tabular}

Solving the matrix using Tora Equation Solver yields;

$V_{1}=4,278.05, V_{2}=15,029.77, V_{3}=23,688.8, V_{4}=6,220.14, V_{5}=447.85$

Table 24 shows a total of 49,665 vehicles for the cumulative commuters of 439,921 as against 85,950 vehicles for 439,916 commuters in Table 21 . This amounts to a reduction of 36,285 vehicles or $42.22 \%$ in passenger traffic volume.

On the other hand, Table 25 shows exhaust emissions of 10,219,880 ppm of Carbon monoxide, 20,998ppm of Nitrogen dioxide and 91,312,000ppm of Methane as against 17,005,800ppm, 41,992ppm and 182,620,000ppm respectively in Table 23. These amount to $40 \%$ reduction in Carbon monoxide, 50\% reduction in Nitrogen dioxide and 50\% reduction in Methane, of exhaust emissions due to passenger vehicles. 
Table 24 Total Commuters provided for by the New Scheme

\begin{tabular}{|l|c|c|c|}
\hline Veh. Types & Number of Vehicle & Weighted Capacity & Total \\
\hline Saloon Veh. $V_{1}$ & 4,278 & 3 & 12,834 \\
\hline Wagon Veh. $V_{2}$ & 15,050 & 5 & 75,150 \\
\hline Mini Buses $V_{3}$ & 23,689 & 9 & 213,201 \\
\hline Coaster Buses $V_{4}$ & 6,220 & 20 & 124,400 \\
\hline Big Buses $V_{5}$ & 448 & 32 & 14,336 \\
\hline Total & 49,665 & & 439,921 \\
\hline
\end{tabular}

Table 25 Total Pollutants Emission by the New Scheme

\begin{tabular}{|c|c|c|c|c|c|c|c|}
\hline \multirow[t]{2}{*}{ Veh. Type } & \multirow{2}{*}{$\begin{array}{l}\text { Total } \\
\text { No. }\end{array}$} & \multicolumn{2}{|c|}{ Carbon Monoxide } & \multicolumn{2}{|c|}{ Nitrogen Dioxide } & \multicolumn{2}{|c|}{ Methane } \\
\hline & & Rate & $\begin{array}{l}\text { Total } \\
\times 10^{3}\end{array}$ & Rate & $\begin{array}{l}\text { Total } \\
\times 10^{3}\end{array}$ & Rate & $\begin{array}{l}\text { Total } \\
\times 10^{6}\end{array}$ \\
\hline Saloon Cars $V_{1}$ & 4,278 & 234.3 & 1002.335 & 0.49 & 2.096 & 4229 & 18.092 \\
\hline Wagon Veh. $V_{2}$ & 15,030 & 158.97 & 2389.32 & 0.49 & 7.365 & 81.67 & 1.228 \\
\hline Mini Buses $V_{3}$ & 23,689 & 225.93 & $5,352.056$ & 0.22 & 5.212 & 2923.33 & 69.251 \\
\hline Coaster Buses $V_{4}$ & 6,220 & 234.30 & $1,457.35$ & 0.63 & 3.919 & 439.67 & 69.251 \\
\hline Big Buses $V_{5}$ & 448 & 42 & 18.816 & 5.37 & 2.406 & 12.67 & 2.735 \\
\hline Total & 49,665 & & $\begin{array}{l}10,219.88 \\
X 10^{3}\end{array}$ & & $\begin{array}{c}20.998 \\
X 10^{3}\end{array}$ & & $\begin{array}{l}91.312 \\
\times 10^{6}\end{array}$ \\
\hline
\end{tabular}

\section{Discussion}

From Table 21 the existing passenger vehicle mix is 34,101:38,368: 11,921: 955: 607, totaling 85,950, that is Salon Vehicles: Wagon vehicles: Mini Buses: Coaster Buses: Big Buses, for a total of 439,916 commuters, The ratio can be written as $56.2: 63.7: 19.6: 1.6: 1$.

It also shows a very high volume of 72,469 vehicles out of 85,950 or $84.3 \%$ of low-passenger carrying capacity vehicles (salons and wagons). From Table 23 these low-passenger carrying vehicles emit into the environment;

$14,089.22 \times 10^{3} \mathrm{ppm}$ or $82.7 \%$ of the Carbon Monoxide

$35.51 \times 10^{3} \mathrm{ppm}$ or $84.6 \%$ of the Nitrogen dioxide and

$147.34 \times 10^{6} \mathrm{ppm}$ or $80.7 \%$ of the Methane.

This scenario of carrying a little but releasing high pollutants resulting in the twin problems of traffic congestion on the roads and highly polluting the environment is very typical in Nigerian Cities, and could be avoided if the new scheme is adopted.

From Table 24 it be observed that the new scheme arrived at has the following corresponding ratio;

4,278: 15, $050: 23,689: 6,220: 448$ for 439,921commuters (a little more), which is $9.5: 33.6: 52.9: 13.9: 1$ 
This is now tilted to favour the high-passenger-carrying vehicles while taking into account pollutants release by them. In the new scheme, the low-passenger-carrying vehicles now total only 19,328 vehicles out of 49,665 amounting to $38.9 \%$.

In the new scheme, the low-passenger-carrying vehicles now emit to the atmosphere

$3,391.66 \times 10^{3} \mathrm{ppm}$ or $33.2 \%$ Carbon monoxide

9. $461 \times 10^{3}$ ppm or $45.1 \%$ of Carbon monoxide and

$19.32 \times 10^{6} \mathrm{ppm}$ or $21.2 \%$ of Methane.

The good attribute of the new scheme is that vehicles of high-passenger-carrying capacities release greater percentage of the pollutants. In the typical case the buses with a combined capacity of 351,937 , which is $80 \%$ of total demand of 439,921 , will emit $66.8 \%$ of carbon monoxide, $54.9 \%$ of Nitrogen dioxide and $78.8 \%$ Methane.

Moreover, the new scheme reduces total traffic volume by 36,285 vehicles from 85,950 to 49,665 representing $42.2 \%$, for a little more commuter.

Also, the new scheme as in Table 25 will result in exhaust emissions of 10,219,880 ppm of Carbon monoxide, 20,998ppm of Nitrogen dioxide and 91,312,000ppm of Methane as against 17,005,800ppm, 41,992ppm and 182,620,000ppm respectively in Table 4.23 . This amounts to $40 \%$ reduction in Carbon Monoxide, $50 \%$ reduction in Nitrogen dioxide and $50 \%$ reduction in Methane, in exhaust emissions due to passenger vehicles.

\subsection{Derived Mix Ratio}

The new scheme suggests a vehicle mix of

$9.5: 33.6: 52.9: 13.9: 1$ of

Salons: Wagons: Mini Buses: Coaster Buses: Big Buses

making a mix total of 110.9 approximately 111

The mix ratio of

$10: 33: 53: 14: 1=111$

can therefore be adopted for commuter vehicles in Owerri.

The result means that for Owerri the ratio of 10:33:53: 14:1 of Salon vehicles, wagon vehicles, Mini buses, Coaster buses and Big buses is optimal for commuters' need and for a minimum carbon monoxide, nitrogen dioxide and methane emission into the environment.

\section{Conclusion}

This work set out to provide a strategy for the management of air pollution owing to road transportation in Owerri Municipal. The result of the field work showed existing commuter vehicles mix of 56.2:63.7: 19.6:1.6:1 of salons, wagons, mini-buses, coaster buses and big buses respectively, of a total of 85,950 vehicles and air ambient pollutants level higher than the recommended standards.

The model developed achieved a remix of 10:33:53:14:1 of same vehicular types and reduction in traffic volume and target air pollutants of about 40-50 percent. Recommendations have been made on economic and regulatory policies that will enable the achievement of the designed vehicular remix.

\section{Recommendations}

By adopting vehicle remix approach, the total number of vehicles was reduced from 85,950 to 49,665 representing a $42.2 \%$, and pollutants release of between $40 \%$ and $50 \%$. 
It is therefore recommended that vehicular remix of 10:33:53:14:1, for salon: wagon: mini-buses: coaster buses: big buses be adopted for Owerri commuters transportation system. In order to achieve this, it is recommended that the state government should adopt economic instruments embedded in a "push and pull" strategy, leveraging on disincentive and incentive measures. Specifically governments should;

- deliberately increase licensing fees for salons and wagons

- introduce bus lanes on major roads (Orlu, Okigwe, Wethedral, Asuumpta, Egbu, Douglas), which gives access to buses only thus reducing their trip time

- introduce equal toll fees on city roads for salons, wagon, and buses

- introduce annual parking fees for cars and wagons.

- encourage private-private or public-private, cooperations in the public bus transportation system with grants.

- have the political will-power, while sensitizing the populace on the need for a sustainable transportation policy, to carry-on with instruments afore listed.

In addition, governments should consider a regulatory policy of outright ban of such low-carrying capacities vehicles like salons and wagons for commercial purposes particularly on some major roads where bus lanes have been suggested.

\section{Compliance with ethical standards}

\section{Disclosure of conflict of interest}

No conflict of interest statement.

\section{References}

[1] Abam FI, Unachukwu GO. Vehicular Emissions and Air Quality Standards in Nigeria. 2009.

[2] European Journal of Scientific Research; Euro Journals Publishing Inc.

[3] ASTM International, https://en.m.wikipedia.ng

[4] Encyclopaedia Britanica (2009), Owerri, Nigeria Retrieved from hhtps://www.britanica.com/place/owerri (2019).

[5] Energy Commission of Nigeria. National Energy Policy, Energy Commission of Nigeria FRN. 2003.

[6] https://www.wisdomjobs.com Solving LP Problems Using Computer with TORA-Quantitative

[7] Technology for Management.

[8] Subramani T. Study of Air Pollution due to Vehicle Emission in Tourism Centre, International Journal of Engineering Research and Applications. 2012; 2(3): 1753-1763.

[9] US EPA. What you can Do to Reduce Pollution from Vehicles and Engines, Transportation, Air Pollution and Climate Change US EPA. 2017.

[10] Zavala MSC, Hemdon EC, Wood JT, Jayne DD, Nelson AM, Trimborn E, Duulea WB, Kinghton A, Mendoza DT, Allen CE, Kolb MJ, Molina, LT Malina. Comparison of \emission from On-Road Sources Using a Mobile Laboratory Under Various Driving and Operational Sampling Msodes, Copernions Publications on behalf of Europan Geosciences Union. 2009. 